\title{
Image Scaling Based on the Catmull-Rom Spline Surfaces with Free Parameters
}

\author{
Juncheng $\mathrm{Li}^{*}$, Lian Yang and Yuee Zhong \\ College of Mathematics and Finance, Hunan University of Humanities, Science and Technology, Loudi 417000, China \\ ${ }^{*}$ Corresponding author
}

\begin{abstract}
This paper presents a class of Catmull-Rom spline surfaces with free parameters, and gives the method for image scaling by using the proposed spline surfaces. The proposed spline surfaces satisfy $C^{2}$ continuity and can be adjusted by the two free parameters even if the data points are kept unchanged. Due to the $C^{2}$ continuity and the two free parameters of the proposed spline surfaces, the details of the original images can be reflected better and the characteristics of the target images could be properly adjusted.
\end{abstract}

Keywords-Catmull-Rom spline; free parameter, interpolation; image scaling

\section{INTRODUCTION}

As we know, interpolation methods are usually used to deal with image scaling problems. The general interpolation methods for image scaling include the nearest interpolation, the bilinear interpolation, the bicubic interpolation and the cubic spline interpolation [1]. Since a 2D grey image can be represented by a continuous function with two variables, the geometric modeling method also can be used to handle the problems of image scaling. In [2], an image scaling method based on the hyperbolic Coons interpolation surface was presented. In [3], the image scaling by using the trigonometric interpolation spline surface was investigated.

Actually, the geometric modeling methods have made great progress. Recently, the curves or surfaces with free parameters have been paid attention by some researchers in geometric modeling, such as the Bézier curves or surfaces with free parameters [4-6] and the B-spline curves or surfaces with free parameters [7-9]. Due to the free parameters, those curves or surfaces with free parameters have better performance ability than the corresponding standard curves or surfaces.

As a method for constructing interpolation curves or surfaces, the cubic Catmull-Rom spline [10] has been widely used in geometric modeling. However, shape of the cubic Catmull-Rom spline can not be adjusted when the data points are fixed, which limits its applications to some extend [11]. The main purpose of this paper is to present a class of Catmull-Rom spline surfaces with free parameters, and then discuss the application of the proposed spline surfaces in image scaling.

\section{THE SPLINE SURFACES}

Firstly, we define the quintic Catmull-Rom basis functions as follows.
Definition 1. For $0 \leq t \leq 1, \alpha \in \mathbb{R}$, the following four functions about $t$ are called the quintic Catmull-Rom basis functions with a free parameter $\alpha$,

$$
\left\{\begin{array}{l}
f_{0}(t)=\frac{1}{2}\left(-t+(3-2 \alpha) t^{2}-(3-6 \alpha) t^{3}+(1-6 \alpha) t^{4}+2 \alpha t^{5}\right), \\
f_{1}(t)=\frac{1}{2}\left(2-(6-4 \alpha) t^{2}+(5-14 \alpha) t^{3}-(1-16 \alpha) t^{4}-6 \alpha t^{5}\right), \\
f_{2}(t)=\frac{1}{2}\left(t+(3-2 \alpha) t^{2}-(1-10 \alpha) t^{3}-(1+14 \alpha) t^{4}+6 \alpha t^{5}\right), \\
f_{3}(t)=\frac{1}{2}\left(-(1+2 \alpha) t^{3}+(1+4 \alpha) t^{4}-2 \alpha t^{5}\right) .
\end{array}\right.
$$

By a simple deduction, the quintic Catmull-Rom basis functions expressed in (1) satisfy that

$$
\left\{\begin{array}{l}
f_{0}(0)=f_{2}(0)=f_{3}(0)=0, \quad f_{1}(0)=1 \\
f_{0}(1)=f_{1}(1)=f_{3}(1)=0, \quad f_{2}(1)=1 .
\end{array}\right.
$$

$$
\left\{\begin{array}{l}
f_{0}^{\prime}(0)=-0.5, \quad f_{1}^{\prime}(0)=f_{3}^{\prime}(0)=0, \quad f_{2}^{\prime}(0)=0.5, \\
f_{0}^{\prime}(1)=f_{2}^{\prime}(1)=0, \quad f_{1}^{\prime}(1)=-0.5, \quad f_{3}^{\prime}(1)=0.5 .
\end{array}\right.
$$

$$
\left\{\begin{array}{l}
f_{0}^{\prime \prime}(0)=3-2 \alpha, \quad f_{1}^{\prime \prime}(0)=-6+4 \alpha, \quad f_{2}^{\prime \prime}(0)=3-2 \alpha, \quad f_{3}^{\prime \prime}(0)=0, \\
f_{0}^{\prime \prime}(1)=0, \quad f_{1}^{\prime \prime}(1)=3-2 \alpha, \quad f_{2}^{\prime \prime}(1)=-6+4 \alpha, \quad f_{3}^{\prime \prime}(1)=3-2 \alpha .
\end{array}\right.
$$

On base of the quintic Catmull-Rom basis functions, we can define the corresponding quintic Catmull-Rom spline surfaces as follows.

Definition 2. Given data points $\boldsymbol{b}_{k, l} \in \mathbb{R}^{3} \quad(k=0,1, \cdots, m$; $l=0,1, \cdots, n)$, for $0 \leq u, v \leq 1, \alpha, \beta \in \mathbb{R}$, the piecewise surfaces

$$
\boldsymbol{p}_{i, j}(u, v)=\sum_{k=0}^{3} \sum_{l=0}^{3} f_{k}(u) f_{l}(v) \boldsymbol{b}_{i+k, j+l}
$$

are called the quintic Catmull-Rom spline surfaces with free parameters $\alpha$ and $\beta$, where $i=0,1, \cdots, m-3 ; j=0,1, \cdots, n-3$, 
$f_{k}(u)$ and $f_{l}(v)(k, l=0,1,2,3)$ are the quintic Catmull-Rom basis functions satisfying that

$$
\begin{aligned}
& \left\{\begin{array}{l}
f_{0}(u)=\frac{1}{2}\left(-u+(3-2 \alpha) u^{2}-(3-6 \alpha) u^{3}+(1-6 \alpha) u^{4}+2 \alpha u^{5}\right), \\
f_{1}(u)=\frac{1}{2}\left(2-(6-4 \alpha) u^{2}+(5-14 \alpha) u^{3}-(1-16 \alpha) u^{4}-6 \alpha u^{5}\right), \\
f_{2}(u)=\frac{1}{2}\left(u+(3-2 \alpha) u^{2}-(1-10 \alpha) u^{3}-(1+14 \alpha) u^{4}+6 \alpha u^{5}\right), \\
f_{3}(u)=\frac{1}{2}\left(-(1+2 \alpha) u^{3}+(1+4 \alpha) u^{4}-2 \alpha u^{5}\right) .
\end{array}\right. \\
& \left\{\begin{array}{l}
f_{0}(v)=\frac{1}{2}\left(-v+(3-2 \beta) v^{2}-(3-6 \beta) v^{3}+(1-6 \beta) v^{4}+2 \beta v^{5}\right), \\
f_{1}(v)=\frac{1}{2}\left(2-(6-4 \beta) v^{2}+(5-14 \beta) v^{3}-(1-16 \beta) v^{4}-6 \beta v^{5}\right), \\
f_{2}(v)=\frac{1}{2}\left(u+(3-2 \beta) v^{2}-(1-10 \beta) v^{3}-(1+14 \beta) v^{4}+6 \beta v^{5}\right), \\
f_{3}(v)=\frac{1}{2}\left(-(1+2 \beta) v^{3}+(1+4 \beta) v^{4}-2 \beta v^{5}\right) .
\end{array}\right.
\end{aligned}
$$

Theorem 1. The quintic Catmull-Rom spline surfaces defined in (5) have the following properties,

(a) Interpolation property: The surfaces interpolate all the given data points expect $\boldsymbol{b}_{0, l}, \boldsymbol{b}_{m, l}(l=0,1, \cdots, n)$ and $\boldsymbol{b}_{k, 0}, \boldsymbol{b}_{k, n}$ $(k=0,1, \cdots, m)$.

(b) $C^{2}$ continuity: The surfaces are $C^{2}$.

(c) Shape adjustable ability: Shapes of the surfaces can be adjusted by the two free parameters $\alpha$ and $\beta$ even if all the data points are kept unchanged.

Proof. (a) From (2) and (5), we have

$$
\begin{cases}\boldsymbol{p}_{i, j}(0,0)=\boldsymbol{b}_{i+1, j+1}, & \boldsymbol{p}_{i, j}(0,1)=\boldsymbol{b}_{i+1, j+2}, \\ \boldsymbol{p}_{i, j}(1,0)=\boldsymbol{b}_{i+2, j+1}, & \boldsymbol{p}_{i, j}(1,1)=\boldsymbol{b}_{i+2, j+2} .\end{cases}
$$

Thus, the surfaces $\boldsymbol{p}_{i, j}(u, v)(i=0,1, \cdots, m-3 ; j=0,1, \cdots, n-3)$ interpolate all the given data points except $\boldsymbol{b}_{0, l}, \boldsymbol{b}_{m, l}$ $(l=0,1, \cdots, n)$ and $\boldsymbol{b}_{k, 0}, \boldsymbol{b}_{k, n}(k=0,1, \cdots, m)$.

(b) From (3), (4) and (5), we have

$$
\left\{\begin{array}{l}
\frac{\partial \boldsymbol{p}_{i, j}(0, v)}{\partial u}=\frac{1}{2} \sum_{l=0}^{3} f_{l}(v)\left(\boldsymbol{b}_{i+2, j+l}-\boldsymbol{b}_{i, j+l}\right), \\
\frac{\partial \boldsymbol{p}_{i, j}(1, v)}{\partial u}=\frac{1}{2} \sum_{l=0}^{3} f_{l}(v)\left(\boldsymbol{b}_{i+3, j+l}-\boldsymbol{b}_{i+1, j+l}\right), \\
\frac{\partial \boldsymbol{p}_{i, j}(u, 0)}{\partial u}=\sum_{k=0}^{3} f_{k}^{\prime}(u) \boldsymbol{b}_{i+k, j+1}, \\
\frac{\partial \boldsymbol{p}_{i, j}(u, 1)}{\partial u}=\sum_{k=0}^{3} f_{k}^{\prime}(u) \boldsymbol{b}_{i+k, j+2} .
\end{array}\right.
$$

$$
\left\{\begin{array}{l}
\frac{\partial \boldsymbol{p}_{i, j}(0, v)}{\partial v}=\sum_{l=0}^{3} f_{l}^{\prime}(v) \boldsymbol{b}_{i+1, j+l}, \\
\frac{\partial \boldsymbol{p}_{i, j}(1, v)}{\partial v}=\sum_{l=0}^{3} f_{l}^{\prime}(v) \boldsymbol{b}_{i+2, j+l}, \\
\frac{\partial \boldsymbol{p}_{i, j}(u, 0)}{\partial v}=\frac{1}{2} \sum_{k=0}^{3} f_{k}(u)\left(\boldsymbol{b}_{i+k, j+2}-\boldsymbol{b}_{i+k, j}\right), \\
\frac{\partial \boldsymbol{p}_{i, j}(u, 1)}{\partial v}=\frac{1}{2} \sum_{k=0}^{3} f_{k}(u)\left(\boldsymbol{b}_{i+k, j+3}-\boldsymbol{b}_{i+k, j+1}\right) .
\end{array}\right.
$$

$$
\left\{\begin{array}{l}
\frac{\partial^{2} \boldsymbol{p}_{i, j}(0, v)}{\partial u \partial v}=\frac{1}{2} \sum_{l=0}^{3} f_{l}^{\prime}(v)\left(\boldsymbol{b}_{i+2, j+l}-\boldsymbol{b}_{i, j+l}\right), \\
\frac{\partial^{2} \boldsymbol{p}_{i, j}(1, v)}{\partial u \partial v}=\frac{1}{2} \sum_{l=0}^{3} f_{l}^{\prime}(v)\left(\boldsymbol{b}_{i+3, j+l}-\boldsymbol{b}_{i+1, j+l}\right), \\
\frac{\partial^{2} \boldsymbol{p}_{i, j}(u, 0)}{\partial u \partial v}=\frac{1}{2} \sum_{k=0}^{3} f_{k}^{\prime}(u)\left(\boldsymbol{b}_{i+k, j+2}-\boldsymbol{b}_{i+k, j}\right), \\
\frac{\partial^{2} \boldsymbol{p}_{i, j}(u, 1)}{\partial u \partial v}=\frac{1}{2} \sum_{k=0}^{3} f_{k}^{\prime}(u)\left(\boldsymbol{b}_{i+k, j+3}-\boldsymbol{b}_{i+k, j+1}\right) .
\end{array}\right.
$$

$$
\left\{\begin{array}{l}
\frac{\partial^{2} \boldsymbol{p}_{i, j}(0, v)}{\partial u^{2}}=(3-2 \alpha) \sum_{l=0}^{3} f_{l}(v)\left(\boldsymbol{b}_{i, j+l}-2 \boldsymbol{b}_{i+1, j+l}+\boldsymbol{b}_{i+2, j+l}\right), \\
\frac{\partial^{2} \boldsymbol{p}_{i, j}(1, v)}{\partial u^{2}}=(3-2 \alpha) \sum_{l=0}^{3} f_{l}(v)\left(\boldsymbol{b}_{i+1, j+l}-2 \boldsymbol{b}_{i+2, j+l}+\boldsymbol{b}_{i+3, j+l}\right), \\
\frac{\partial^{2} \boldsymbol{p}_{i, j}(u, 0)}{\partial u^{2}}=\sum_{k=0}^{3} f_{k}^{\prime \prime}(u) \boldsymbol{b}_{i+k, j+1}, \\
\frac{\partial^{2} \boldsymbol{p}_{i, j}(u, 1)}{\partial u^{2}}=\sum_{k=0}^{3} f_{k}^{\prime \prime}(u) \boldsymbol{b}_{i+k, j+2} .
\end{array}\right.
$$

$$
\left\{\begin{array}{l}
\frac{\partial^{2} \boldsymbol{p}_{i, j}(0, v)}{\partial v^{2}}=\sum_{l=0}^{3} f_{l}^{\prime \prime}(v) \boldsymbol{b}_{i+1, j+l}, \\
\frac{\partial^{2} \boldsymbol{p}_{i, j}(1, v)}{\partial v^{2}}=\sum_{l=0}^{3} f_{l}^{\prime \prime}(v) \boldsymbol{b}_{i+2, j+l}, \\
\frac{\partial^{2} \boldsymbol{p}_{i, j}(u, 0)}{\partial v^{2}}=(3-2 \beta) \sum_{k=0}^{3} f_{k}(u)\left(\boldsymbol{b}_{i+k, j}-2 \boldsymbol{b}_{i+k, j+1}+\boldsymbol{b}_{i+k, j+2}\right), \\
\frac{\partial^{2} \boldsymbol{p}_{i, j}(u, 1)}{\partial v^{2}}=(3-2 \beta) \sum_{k=0}^{3} f_{k}(u)\left(\boldsymbol{b}_{i+k, j+1}-2 \boldsymbol{b}_{i+k, j+2}+\boldsymbol{b}_{i+k, j+3}\right) .
\end{array}\right.
$$

Expression (6) shows that

$$
\begin{cases}\boldsymbol{p}_{i, j}(1,0)=\boldsymbol{p}_{i+1, j}(0,0), & \boldsymbol{p}_{i, j}(1,1)=\boldsymbol{p}_{i+1, j}(0,1), \\ \boldsymbol{p}_{i, j}(0,1)=\boldsymbol{p}_{i, j+1}(0,0), & \boldsymbol{p}_{i, j}(1,1)=\boldsymbol{p}_{i, j+1}(1,0)\end{cases}
$$

Expressions (7) and (8) show that 


$$
\begin{cases}\frac{\partial \boldsymbol{p}_{i, j}(1, v)}{\partial u}=\frac{\partial \boldsymbol{p}_{i+1, j}(0, v)}{\partial u}, & \frac{\partial \boldsymbol{p}_{i, j}(u, 1)}{\partial u}=\frac{\partial \boldsymbol{p}_{i, j+1}(u, 0)}{\partial u}, \\ \frac{\partial \boldsymbol{p}_{i, j}(1, v)}{\partial v}=\frac{\partial \boldsymbol{p}_{i+1, j}(0, v)}{\partial v}, & \frac{\partial \boldsymbol{p}_{i, j}(u, 1)}{\partial v}=\frac{\partial \boldsymbol{p}_{i, j+1}(u, 0)}{\partial v} .\end{cases}
$$

Expressions (9), (10) and (11) show that

$$
\begin{cases}\frac{\partial^{2} \boldsymbol{p}_{i, j}(1, v)}{\partial u \partial v}=\frac{\partial^{2} \boldsymbol{p}_{i+1, j}(0, v)}{\partial u \partial v}, & \frac{\partial^{2} \boldsymbol{p}_{i, j}(u, 1)}{\partial u \partial v}=\frac{\partial^{2} \boldsymbol{p}_{i, j+1}(u, 0)}{\partial u \partial v}, \\ \frac{\partial^{2} \boldsymbol{p}_{i, j}(1, v)}{\partial u^{2}}=\frac{\partial^{2} \boldsymbol{p}_{i+1, j}(0, v)}{\partial u^{2}}, & \frac{\partial^{2} \boldsymbol{p}_{i, j}(u, 1)}{\partial u^{2}}=\frac{\partial^{2} \boldsymbol{p}_{i, j+1}(u, 0)}{\partial u^{2}}, \\ \frac{\partial^{2} \boldsymbol{p}_{i, j}(1, v)}{\partial v^{2}}=\frac{\partial^{2} \boldsymbol{p}_{i+1, j}(0, v)}{\partial v^{2}}, & \frac{\partial^{2} \boldsymbol{p}_{i, j}(u, 1)}{\partial v^{2}}=\frac{\partial^{2} \boldsymbol{p}_{i, j+1}(u, 0)}{\partial v^{2}} .\end{cases}
$$

According to (12), (13) and (14), we can obtain that the surfaces are $C^{2}$.

(c) Since (5) contains two free parameters $\alpha$ and $\beta$, we could obtain different shapes of surfaces for different values of $\alpha$ and $\beta$ when all the data points are fixed. $\square$

According to the interpolation property and $C^{2}$ continuity of the quintic Catmull-Rom spline surfaces, if we add auxiliary points $\boldsymbol{b}_{-1, j}, \quad \boldsymbol{b}_{m+1, j}(j=-1,0, \cdots, n+1)$ and $\boldsymbol{b}_{i,-1}$, $\boldsymbol{b}_{i, n+1}(i=0,1, \cdots, m)$ to the given data points $\boldsymbol{b}_{k, l}(k=0,1, \cdots, m$; $l=0,1, \cdots, n)$, the $C^{2}$ quintic Catmull-Rom spline surfaces interpolating all the given data points would be generated. Generally, the auxiliary points can be added as follows,

$$
\left\{\begin{array}{l}
\boldsymbol{b}_{-1,-1}=2 \boldsymbol{b}_{0,0}-\boldsymbol{b}_{1,1}, \quad \boldsymbol{b}_{-1, n+1}=2 \boldsymbol{b}_{0, n}-\boldsymbol{b}_{1, n-1}, \\
\boldsymbol{b}_{m+1,-1}=2 \boldsymbol{b}_{m, 0}-\boldsymbol{b}_{m-1,1}, \quad \boldsymbol{b}_{m+1, n+1}=2 \boldsymbol{b}_{m, n}-\boldsymbol{b}_{m-1, n-1}, \\
\boldsymbol{b}_{-1, j}=2 \boldsymbol{b}_{0, j}-\boldsymbol{b}_{1, j}, \quad \boldsymbol{b}_{m+1, j}=2 \boldsymbol{b}_{m, j}-\boldsymbol{b}_{m-1, j}, \quad j=0,1, \cdots, n, \\
\boldsymbol{b}_{i,-1}=2 \boldsymbol{b}_{i, 0}-\boldsymbol{b}_{i, 1}, \quad \boldsymbol{b}_{i, n+1}=2 \boldsymbol{b}_{i, n}-\boldsymbol{b}_{i, n-1}, \quad i=0,1, \cdots, m .
\end{array}\right.
$$

From Theorem 1, the quintic Catmull-Rom spline surfaces not only inherit the interpolation property and continuity of the standard cubic Catmull-Rom spline surfaces [10], but also have $C^{2}$ continuity and shape adjustable ability. When the data points and auxiliary points are kept unchanged, shapes of the the quintic Catmull-Rom spline surfaces can be adjusted by the two free parameters $\alpha$ and $\beta$. Figure 1 shows the standard cubic Catmull-Rom spline surfaces and the quintic CatmullRom spline surfaces for different values of $\alpha$ and $\beta$ generated by nine patches, where (a) shows the standard cubic CatmullRom spline surfaces, (b), (c) and (d) shows the quintic Catmull-Rom spline surfaces for $(\alpha, \beta)=(-1,3),(\alpha, \beta)=(3,7)$ and $(\alpha, \beta)=(9,5)$ respectively.

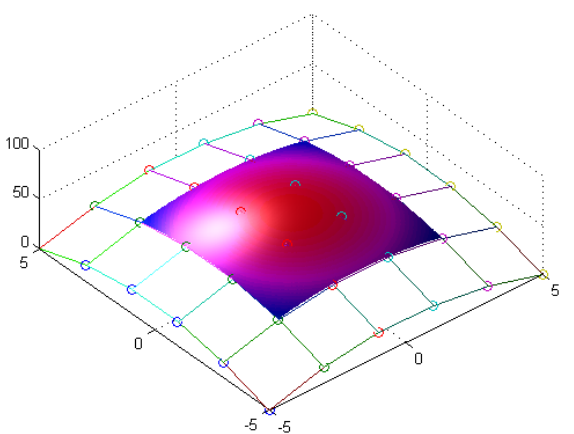

(a)

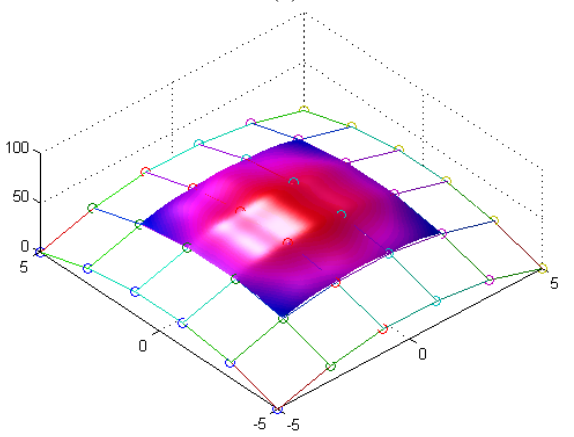

(b)

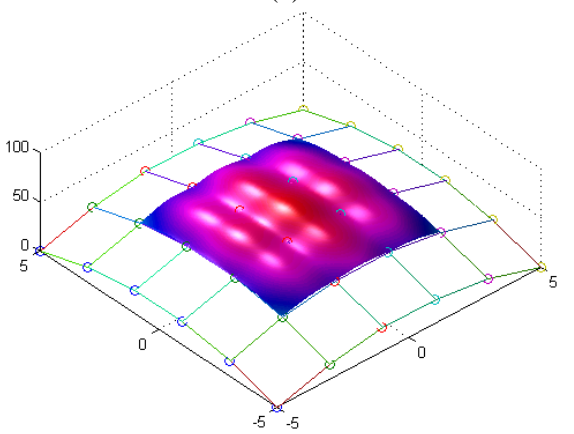

(c)

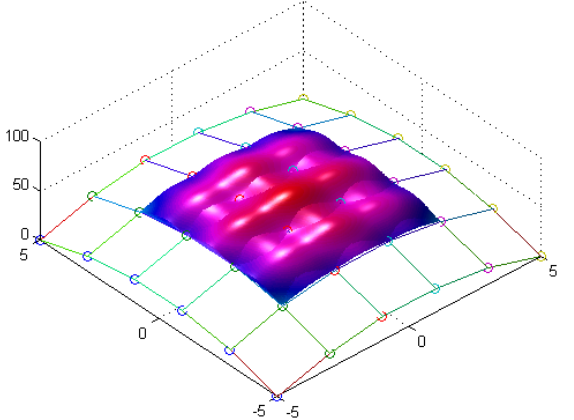

(d)

FIGURE I. THE STANDARD CUBIC CATMULL-ROM AND THE QUINTIC CATMULL-ROM SPLINE SURFACES

\section{IMAGE SCALING METHOD}

Given a gray image $I(x, y)$ with the size of $M \times N$, the method for enlarging the original image $I(x, y)$ into the target image $I^{\prime}(x, y)$ with the size of $M_{1} \times N_{1}$, based on surface interpolation, roughly includes the following two steps [2], 
Step1. A surface $F(x, y)(0 \leq x \leq M-1,0 \leq y \leq N-1)$ interpolating all the pixel points of the original image $I(x, y)$ needs to be constructed.

Step2. The target image $I^{\prime}(x, y)$ would be gotten by resampling the interpolation surface $F(x, y)$ according to the scaling ratio.

According to (5), we can express the functions of the quintic Catmull-Rom surfaces as follows,

$$
\begin{aligned}
& p_{i, j}(u, v)=\left(f_{0}(u) \quad f_{1}(u) \quad f_{2}(u) \quad f_{3}(u)\right) \times \\
& \left(\begin{array}{cccc}
b_{i, j} & b_{i, j+1} & b_{i, j+2} & b_{i, j+3} \\
b_{i+1, j} & b_{i+1, j+1} & b_{i+1, j+2} & b_{i+1, j+3} \\
b_{i+2, j} & b_{i+2, j+1} & b_{i+2, j+2} & b_{i+2, j+3} \\
b_{i+3, j} & b_{i+3, j+1} & b_{i+3, j+2} & b_{i+3, j+3}
\end{array}\right)\left(\begin{array}{l}
f_{0}(v) \\
f_{1}(v) \\
f_{2}(v) \\
f_{3}(v)
\end{array}\right) .
\end{aligned}
$$

Let $g_{i, j}(i=0,1, \cdots, M-1 ; j=0,1, \cdots, N-1)$ are the gray values of the original image $I(x, y), g_{i_{1}, j_{1}}^{\prime}\left(i_{1}=0,1, \cdots, M_{1}-1\right.$; $\left.j_{1}=0,1, \cdots, N_{1}-1\right)$ are the gray values of the target image $I^{\prime}(x, y)$. According to (15), we add the gray values of the pixels around the image $I(x, y)$ as follows,

$$
\left\{\begin{array}{l}
g_{-1,-1}=2 g_{0,0}-g_{1,1}, \quad g_{-1, N}=2 g_{0, N-1}-g_{1, N-2}, \\
g_{M,-1}=2 g_{M-1,0}-g_{M-2,1}, \quad g_{M, N}=2 g_{M-1, N-1}-g_{M-2, N-2}, \\
g_{-1, j}=2 g_{0, j}-g_{1, j}, \quad g_{M, j}=2 g_{M-1, j}-g_{M-2, j}, \quad j=0,1, \cdots, N-1, \\
g_{i,-1}=2 g_{i, 0}-g_{i, 1}, \quad g_{i, N}=2 g_{i, N-1}-g_{i, N-2}, \quad i=0,1, \cdots, M-1 .
\end{array}\right.
$$

Then, refer to [2], the gray value $g_{i_{1}, j_{1}}^{\prime}$ of the target image $I^{\prime}(x, y)$ can be computed as

$$
g_{i_{1}, j_{1}}^{\prime}=p_{i, j}(u, v)
$$

where $u=\frac{M}{M_{1}} i_{1}-\left\lfloor\frac{M}{M_{1}} i_{1}\right\rfloor, v=\frac{N}{N_{1}} j_{1}-\left\lfloor\frac{N}{N_{1}} j_{1}\right\rfloor$.

Due to the $C^{2}$ continuity of the quintic Catmull-Rom spline surfaces, the details of the original image could be reflected better. Moreover, we can properly adjust the characteristics of the target image by altering values of the two free parameters $\alpha$ and $\beta$.

At the end of this section, let us give an experiment to illustrate the effectiveness of the proposed method. For comparing the experiment effects between the proposed method and the method presented in [2], we first reduce the original images 4 times by bilinear interpolation. Then, we use the proposed method and the method presented in [2] to scale the contractible images 4 times respectively. Figure 2 shows the experiment results with the corresponding edge detection, where the free parameters in [2] and in the proposed method are taken as the optimal values that can make the PSNR as high as possible.

From Figure 2, we can see that the proposed method has better image scaling effect than the method presented in [2].

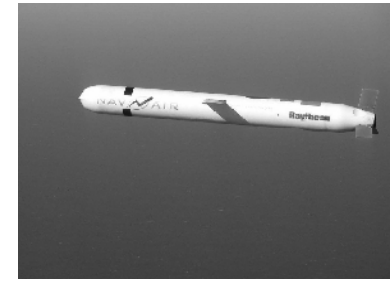

(a) Original image

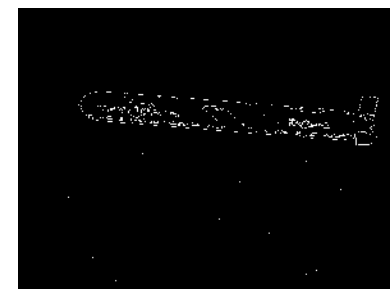

(d) Edge detection of (a)

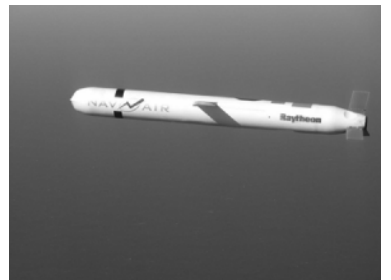

(b) Method in [2]

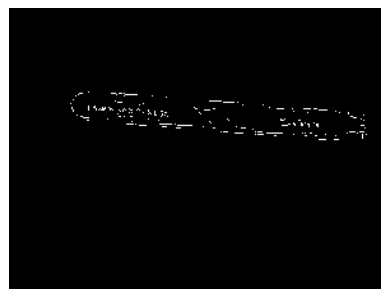

(e) Edge detection of (b)

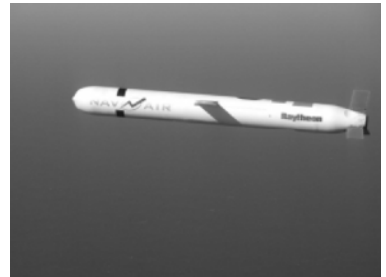

(c) Proposed method

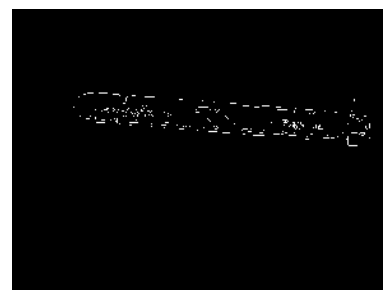

(f) Edge detection of (c)

FIGURE II. EXPERIMENT RESULTS

\section{REFERENCES}

ACKNOWLEDGMENT

This work was supported by the Hunan Provincial Natural Science Foundation of China (2017JJ3124).
[1] K. R. Castleman. Digital Image Processing, Prentice Hall,1995.

[2] J. C. Li. Image enlargement based on the hyperbolic Coons interpolation, Computer Modelling and New Technologies, vol. 18, 2014, pp. 79-85. 
[3] J. C. Li, L. Yang. The trigonometric interpolation spline surface and its application in image zooming, The Seventh International Conference on Digital Image Processing (ICDIP 2015), Los Angeles, United States, July 6, 2015.

[4] L. L. Yan, J. F. Liang. An extension of the Bézier model, Applied Mathematics and Computation, vol. 218, 2011, pp. 2863-2879.

[5] J. C. Li. A class of cubic trigonometric Bézier curve with a shape parameter, Journal of Information and Computational Science, vol. 10, 2013, pp. 3071-3078.

[6] U. Bashir, M. Abbsa, J. M. Ali. The $G^{2}$ and $C^{2}$ rational quadratic trigonometric Bézier curve with two shape parameters with applications, Applied Mathematics and Computation, vol. 219, 2013, pp. 1018310197.

[7] X. M. Liu, W. X. Xu, Y. Guan Yong, et al. Hyperbolic polynomial uniform B-spline curves and surfaces with shape parameter, Graphical Models, vol. 72, 2010, pp. 1-6.

[8] J. Cao, G. Z. Wang. Non-uniform B-spline curves with multiple shape parameters, Journal of Zhejiang University Science C, vol. 12, 2011, pp. 800-808.

[9] L. L. Yan, J. F. Liang. A class of algebraic-trigonometric blended splines. Journal of Computational and Applied Mathematics, vol. 235, 2011, pp. 1713-1729.

[10] T. D. Derose, B. A. Barsky. Geometric continuity, shape parameters, and geometric constructions for Catmull-Rom splines, ACM Transactions on Graphics, vol. 7, 1988, pp. 1-41.

[11] J. C. Li, S. Chen. The cubic $\alpha$-Catmull-Rom spline. Mathematical and Computational Applications, vol. 21, 2016. 